\title{
Article
}

\section{Amyloidosis and cardiovascular diseases: A clinical insight}

\author{
Visser, Renier Adriaan Binnemann, Gravenor, Céline, Ahmed, \\ Sennia and Harky, Amer \\ Available at http://clok.uclan.ac.uk/36019/ \\ Visser, Renier Adriaan Binnemann, Gravenor, Céline, Ahmed, Sennia and \\ Harky, Amer (2021) Amyloidosis and cardiovascular diseases: A clinical \\ insight. Journal of Cardiac Surgery, 36 (2). pp. 522-529. ISSN 0886-0440
}

It is advisable to refer to the publisher's version if you intend to cite from the work. http://dx.doi.org/10.1111/jocs.15230

For more information about UCLan's research in this area go to http://www.uclan.ac.uk/researchgroups/ and search for < name of research Group>.

For information about Research generally at UCLan please go to http://www.uclan.ac.uk/research/

All outputs in CLoK are protected by Intellectual Property Rights law, including Copyright law. Copyright, IPR and Moral Rights for the works on this site are retained by the individual authors and/or other copyright owners. Terms and conditions for use of this material are defined in the policies page.

\section{CLoK}

Central Lancashire online Knowledge www.clok.uclan.ac.uk

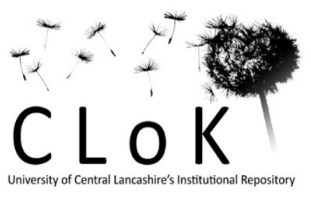


1) P a g e

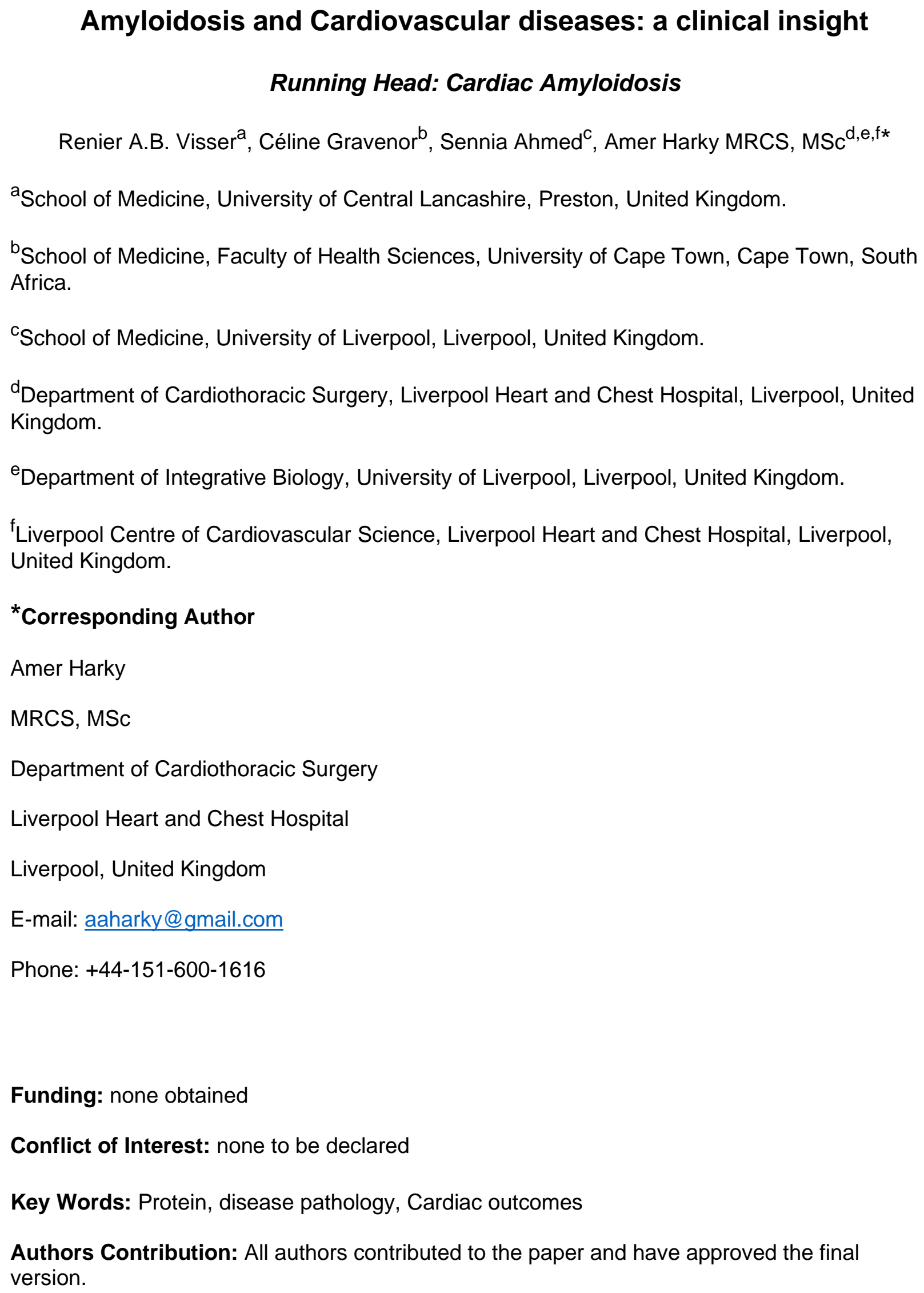


2) P a g e

49

50

51

52

53

54

55

56

57

58

59

60

61

62

\section{Abstract}

Systemic amyloidosis is caused by deposition of amyloid proteins in varying organ systems throughout the body, leading to dysfunction within those systems. The development of cardiac amyloidosis is one of the main indicators for a poor prognosis for patients. Cardiac amyloidosis is most commonly caused by the Immunoglobulin light chain amyloidosis and the transthyretin amyloidosis. Both have poor prognoses when associated with cardiac amyloidosis, however, the patients with the former subtype fair far worse than those with the later. Despite amyloidosis having a history of being underdiagnosed, recent epidemiological data indicates that the rate of diagnosis has increased which has coincided with an improved in patient median survival rates. It is of great importance that patients are diagnosed with the correct subtype as the main treatment strategy is to treat the underlying cause of the amyloidosis. If a misdiagnosis is made patients can receive treatment that might be ineffective or even harmful.

There is a great of progress being made in the pharmacological treatments being made for treating the underlying causes, however, many of the proposed treatments still need more evidence to support its use.

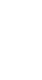

(1)

(1)




\section{Introduction}

Systemic amyloidosis is a rare group of diseases caused by the extracellular deposition of amyloid proteins in various tissues throughout the body ${ }^{1}$. As of yet more than 30 proteins have been found that can aggregate as an amyloid protein, however, with the use of mass spectrometry it is suspected that there are many more of these precursor proteins ${ }^{2}$.

In the majority of the cases, cardiac amyloidosis $(\mathrm{CA})$ is caused by two common subtypes of these proteins, immunoglobulin light chain (AL) amyloidosis and transthyretin (ATTR) amyloidosis, together accounting for approximately $98 \%$ of all cases of $\mathrm{CA}^{1,3-4}$. Not only do AL and ATTR amyloidosis have markedly different prognoses, with a median survival of 6-12 months and 2-6 years respectively, they also have significantly different treatments which is why there is a great emphasis on specifying the subtype of amyloidosis ${ }^{3-5}$.

The main focus of this review will revolve around CA as it is the leading cause of morbidity and mortality in systemic amyloidosis regardless of the subtype ${ }^{1}$. As it has been shown that the prevalence of $\mathrm{CA}$ increases with age, this is of ever greater importance due to the expanding aging population ${ }^{6}$. However, amyloidosis is probably still underdiagnosed and even when diagnosed, the different subtypes with their own specific treatments go unrecognized, which can lead to mismanagement of patients ${ }^{7}$. This emphasises the need of an updated review on CA to familiarise clinicians with the specifics of amyloidosis towards improved patient care ${ }^{2}$.

\section{Pathophysiology}

Amyloidosis is characterised by infiltrative deposits of 'amyloid proteins', which are typically waxy, starch-like deformed protein fibrils ${ }^{7}$. Many different types of causative proteins have been identified, some causing systemic amyloidosis, with deposits in multiple organ systems, and others showing more localised pathology in a specific organ ${ }^{8}$. Some well-known diseases are caused by localised misfolded protein deposits (i.e. localised amyloidosis), such as Alzheimer's 
disease (Amyloid- $\beta$ precursor protein), Creutzfeld-Jakob disease (Prion protein) and familial dementias ${ }^{8}$. Proteins may misfold and form amyloid fibrils if mutations occur in the genetic coding for that protein, causing amino acid substitutions, or if the protein stability is compromised ${ }^{5}$. As with most diseases, factors are hereditary as well as environmental (chemical, electrical, and mechanical stimuli) ${ }^{7}$. This review focuses on the amyloidosis types that are most prominent in causing cardiac complications: immunoglobulin amyloidosis (AL), ATTRwt amyloidosis (formerly called senile systemic amyloidosis [SSA]), ATTRv amyloidosis (formerly called familial amyloid polyneuropathy [FAP]), Table 1 provides a summary of each of the above mentioned subtypes ${ }^{8}$.

\subsection{Immunoglobulin light chain (AL) amyloidosis:}

$\mathrm{AL}$ amyloidosis is characterised by a malignant plasma cell clone with a resulting excess of a particular misfolded light- or heavy-chain immunoglobulin (Ig) which is then systemically deposited $^{8}$. The AL subtype is present in about $15 \%$ of patients with multiple myeloma (MM), however isolated $\mathrm{AL}$ amyloidosis (primary $\mathrm{AL}$ ) has a different pattern of disease and is therefore an alone standing diagnosis ${ }^{8}$. Public Health England classifies primary AL amyloidosis as a cancer, and according to one report, it accounts for $65 \%$ of $5-20$ per million cases of amyloidosis in the $\mathrm{UK}^{9}$. AL amyloidosis can affect multiple organs, or only a single organ, usually the kidney, heart, liver, gut, and peripheral nervous system ${ }^{7-8}$. The onset is rapid and treatment is targeted at the monoclonal immunoglobulin-producing cells ${ }^{8}$. Treatment that reduces the circulating free light chains (FLC) has better outcomes for heart failure, because FLC seems to be toxic to myocardial cells ${ }^{10}$. This suggests that Cardiac-related AL amyloidosis (AL-CA) is "not simply an infiltrative cardiomyopathy but rather a toxic infiltrative disorder"10.

\subsection{Transthyretin (ATTR) Amyloidosis:}

ATTR Amyloidosis caused by misfolded or destabilised transthyretin (TTR) ${ }^{7}$. TTR is produced predominantly by the liver, retinal pigment epithelium and choroid plexus ${ }^{8}$. Two main types of TTR cause CA: a wild-type (AATRwt) and variant-type (ATTRv ${ }^{7-8}$. ATTRwt amyloidosis is 
142 associated with advancing age (hence the former name 'systemic senile amyloidosis'). The

143 hypothesised mechanism of disease is that TTR, a cyclic tetramer protein in circulation,

144 becomes unstable in elderly patients, and is deposited as pathologic amylaceous (starchy)

145 fibrils ${ }^{8}$. The ATTRv subtype is caused by a 'variant' TTR with altered genetic mutation, inherited

146 in an autosomal dominant pattern ${ }^{8}$. The onset may be early, with gradually increasing severity of

147 symptoms over several decades ${ }^{8}$. There are multiple types of mutations, with varying degrees of

148 resultant instability of the TTR tetramer ${ }^{11}$. The resulting amyloid fibrils are deposited in various

149 systems depending on the mutant type; in peripheral and autonomic nerves, heart, gut, kidney,

150 eyes and brain ${ }^{8}$. It was found that mutations associated with greater instability in TTR affected

151 the peripheral nervous and oculo-leptomeningeal systems more frequently, whereas more

152 stable TTR variants showed greater affinity for the cardiac system ${ }^{12}$.

\section{Cardiac Amyloidosis (CA):}

156 Clinical manifestation is dependent upon the type of amyloidosis and the site of its occurrence

157 (Figure 1). The complexity tends to develop due cardiac involvement either as part of a systemic

158 process or a local phenomenon. Surprisingly, it is not the quantity of organ systems affected, but 159 the deposition of amyloid fibrils in the heart that decrees a poor prognosis ${ }^{10}$.

$160 \mathrm{CA}$ is thought to be underdiagnosed ${ }^{13}$. Early recognition and diagnosis are needed to delay the 161 progression of the disease and increase suitability for surgical interventions ${ }^{13}$. CA should be 162 considered as an important differential in patients with heart failure particularly if ejection fraction 163 is preserved $(\mathrm{HFpEF})^{13}$.

164 Amyloid deposits may be found in one or all of the heart layers: endo-, myo- and pericardium ${ }^{13}$.

165 The endo-myocardium will be the focus of this literature review and refers to the lining of the four 166 chambers as well as the valves, and the cardiac muscle itself ${ }^{13}$. Though nodular deposits of 
6|Pag e

167

168

169

170

171

172

173

174

175

176

177 Each subtype of amyloidosis has specific symptoms associated with it which are presented in

amyloid may be found on the pericardium ${ }^{13}$, this will not be discussed any further in this review as it is not discussed at great length in the existing literature.

\subsection{Diagnosing:}

At first glance, diagnosing CA seems relatively straight forward. However, some of the difficulties in reaching a timely diagnosis include the rarity of the disease, late presentation of diverse clinical symptoms and the existence of various subtypes ${ }^{14}$. CA is also often mistaken for hypertrophic cardiomyopathy with left ventricular outflow tract obstruction (LVOTO) ${ }^{7}$. Thus, a surprise encounter on the operating table is not uncommon but significant advancements in diagnostic methods should decrease such scenarios moving forward ${ }^{14}$.

\subsubsection{Clinical:}

Extra cardiac manifestations can be varied depending on the type of amyloid precursor in

Figure 2. CA classically presents as a rapidly progressive congestive heart failure in the absence of ischaemic pathology, which may be accompanied by conduction system disturbances such as arrhythmias and heart blocks ${ }^{15}$.

Congestive heart failure due to restrictive cardiomyopathy should be met with high clinical suspicion ${ }^{15}$. The amyloidotic heart is firm, rubbery and less compliant due to the replacement of myocytes with amyloid deposits ${ }^{15}$. Subsequently cardiac relaxation is impaired, causing an increase in right sided filling pressures. Increased jugular venous pressure, hepatomegaly and peripheral oedema are not uncommon ${ }^{15}$.

Patients may complain of exertional dyspnoea, fatigue, and chest discomfort. In severe cases cardiac cachexia may also be present ${ }^{13,15}$.

question. For instance, in AL periorbital purpura is highly characteristic of CA and should prompt 
190

191

192

a tissue biopsy ${ }^{7}$. A comprehensive history and examination may also suggest leg/jaw claudication, macroglossia and proteinuria ${ }^{7}$.

\subsubsection{Echocardiogram:}

An echocardiogram $(\mathrm{ECHO})$ is very useful in illustrating the morphological changes that occur over time in an amyloidotic heart and has $87 \%$ sensitivity for $\mathrm{CA}^{14}$, however the reliability may vary with the practitioner's familiarity with $\mathrm{CA}$ on $\mathrm{ECHO}$. There is an increase in bi-ventricular and bi-atrial wall thickness, with no change in chamber size and dilatation respectively ${ }^{14}$. The atria undergo dilatation to withstand filling pressures ${ }^{14}$. Septal infiltration and valvular thickening as well as pericardial effusions are also common ${ }^{14}$. The abnormal texture of the infiltrated endomyocardium appears as 'granular and sparkling', due to the discrepancy between the normal myocytes and the shimmering amyloid protein ${ }^{14}$.

\subsubsection{Electrocardiograph:}

Low voltage QRS complexes on an ECG, in combination with the aforementioned positive ECHO findings is highly indicative of CA and is found in $50 \%$ of patients with CA. This is because CA associated ventricular hypertrophy is due to an infiltrative process and not true myocyte hypertrophy as seen in hypertrophic cardiomyopathy ${ }^{16}$. Amyloid deposits are insulative resulting in reduced QRS amplitudes?.

Combining the clinical history, ECHO findings and a discordant ECG underlines the difficulties in diagnosing CA. CA is nothing short of a masquerade and mimics heart failure, hypertrophic cardiomyopathy and ischaemic changes (non-Q wave infarction).

\subsubsection{Biopsy and staining:}


211 Endomyocardial biopsy is not the only means by which tissue can be obtained for immuno-

212 characterisation of amyloid precursors. Extra-cardiac sites, in particular, the abdominal fat pad,

213 are also viable possibilities ${ }^{7,16}$. Aspiration of the subcutaneous fat has successfully helped 214 diagnose CA in $85 \%$ of patients ${ }^{16}$.

215 The gold standard for diagnosing amyloidosis subtypes remains histological staining and 216 immunophenotyping of tissue biopsies ${ }^{7}$. Stains such as methyl violet, thioflavin, and haematoxylin 217 and eosin (H\&E) can be used ${ }^{7}$. The most selected stain is the Congo red which under polarised 218 light, produces an apple-green birefringence 7 . However, it should be noted that abundant collagen 219 on aortic valves can produce a false positive Congo stain, leading to possibly an incorrect 220 diagnosis of $\mathrm{CA}^{14}$. Gertz et al. suggest using mass spectrometry in addition to staining to better 221 guide clinical decisions ${ }^{16}$.

\section{3.1.5 Magnetic resonance imaging $(M R I)$ :}

223 The new MRI "late gadolinium enhancement" technique has been shown to be effective in 224 diagnosing amyloidosis ${ }^{6}$. This is done by identifying a characteristic pattern of increased 225 myocardial enhancement with other morphological findings such as increased left ventricle wall 226 thickening and abnormal myocardial and blood pool kinetics ${ }^{6}$. Other MRI imaging techniques such

227 as long axis strain and myocardial contraction fraction have been shown to have great prognostic 228 value, as well as determining morphological and functional markers of disease ${ }^{17}$. However, more 229 evidence is needed to support the two latter techniques before they become more commonly 230 used $^{17}$.

\section{$231 \quad 3.2$ Staging}

232 The revised Mayo Clinic amyloidosis staging system, in addition to the New York Heart 233 Association (NYHA) four stage system for congestive HF, can be used to determine progression 
234 and severity of $\mathrm{AL}$ amyloidosis ${ }^{5,8}$. In Mayo Clinic staging system patient are assigned a score of

2351 for each difference in FLC (difference between involved and uninvolved FLC) $\geq 18 \mathrm{mg} / \mathrm{dL}$ $236(180 \mathrm{mg} / \mathrm{L}), \mathrm{cTnT} \geq 0.025 \mathrm{ng} / \mathrm{mL}$, and NT-proBNP $\geq 1,800 \mathrm{pg} / \mathrm{mL}$, creating stages I to IV with scores

237 of 0 to 3 points $^{8}$. Patients diagnosed with the Mayo Clinic staging system had rapidly decreasing 238 survival rates with higher stages, and in a cohort treated for CA, those treated earlier on (in stage 239 I-II of NYHA staging) had better responses to treatment ${ }^{18}$.

\section{$240 \quad 3.3$ Diagnostic epidemiological data}

241 As stated above, it is important to diagnose CA early on, and that clinicians are aware of the 242 cardiac and systemic signs, as well as the up-to-date diagnostic techniques for improved patient 243 outcomes. A 2018 epidemiological study in the United States showed an increasing proportion of 244 the population being diagnosed with amyloidosis each year ${ }^{19}$, which suggests increasing 245 awareness of the disease amongst medical professionals. This study also noted increased 246 survival rates, which is reflected by the trends observed by a UK-based epidemiological study in $2472017^{20}$. This shows significant development in management of amyloidosis, and advancement in 248 general management of complex chronic disease in elderly patients.

\section{4. Vascular amyloidosis}

250 The current literature has a small number of case reports on the presence of amyloid deposits in 251 vasculature. Although some attempt is made to understand vascular amyloidosis (VA), there is 252 no transparency regarding the frequency and extent of vascular involvement ${ }^{21-22 .}$

253 Amyloidosis affecting the vasculature can present as myopathy, jaw claudication and/or angina ${ }^{21}$.

254 Microvascular involvement is common and often results in increased serum troponin leading to a 255 misdiagnosis of 'non-Q wave' infarction in the absence of true coronary artery disease ${ }^{13}$. 256 Myocardial flow reserve becomes impaired in VA leading to myocyte necrosis ${ }^{13}$. 
10 | P a g e

257 Amyloid deposits can affect the morphology of the vessel and/or the functionality. The dependent

258 variables are frequently reported as carotid artery intimal thickness (IMT) and brachial artery flow

259 mediated dilatation (FMD) ${ }^{21}$.

\section{$260 \quad 4.1$ Coronary vessels}

261 In a cohort study conducted by Sharma et al. AL amyloidosis was associated with a transmural

262 deposition of amyloid whereas ATTRwt amyloidosis was found mainly in the adventitia and 263 external media ${ }^{22}$. This implies that there is also a distinction to be made on location of deposits

264 and not just the frequency and extent. Coronary vessels were affected in all but one in the patient

265 group. However, it was noted that vessel lumen was significantly affected in AL amyloidosis, likely

266 due to transmural deposit pattern ${ }^{22}$.

267 Smith et al. measured the frequency of vascular involvement (as well as other morphologic 268 markers) in AL amyloidosis compared to ATTRwt amyloidosis ${ }^{23}$. A total of 47 autopsy proven 269 amyloidotic hearts were analysed, $\mathrm{AL}$ amyloidosis showed higher inclination for vasculature (90 $270 \%)$ whereas this figure was $4 \%$ for ATTRwt amyloidosis ${ }^{23}$. Crotty et al found the percentages to 271 be $88 \%$ and $26 \%$ for the AL and ATTRwt subtypes respectively ${ }^{24}$.

272 Dorbala et al. proposed that coronary artery flow can be impeded via three different pathways: A) 273 Deposits in the vessel wall causing stenosis of lumen (structural), B) extrinsic compression due

274 to interstitial deposits and C) endothelial dysfunction ${ }^{25}$. A comparison was made between patients 275 with amyloidosis versus those with hypertrophic cardiomyopathy in relation to coronary 276 perfusion ${ }^{25}$. MBF (myocardial blood flow) and CRF (coronary flow reserve) were reduced in the 277 amyloidosis group suggesting microvascular involvement ${ }^{25}$. 
11 | P a g e

279 Amyloidosis of the aorta is more likely to be found in the ATTRwt subtype ${ }^{26-28}$. It is associated with

280 an increase in age and is thus a manifestation of senile amyloidosis ${ }^{26-28}$. Iwata et al. investigated

281224 ATTRwt amyloidosis autopsy cases and found aortic amyloidosis to an average incidence of

$28279 \%{ }^{27}$. They found that the media of the aorta was affected by amyloidosis, which presented

283 multiple minute deposits and having no relation to atherosclerosis ${ }^{27}$. ATTRwt amyloidosis in the

284 aorta has been suspected of one of the possible cause of aortic aneurysms but this claim still

285 needs to be investigated further ${ }^{28}$. There have been no comprehensive studies to suggest that

286 aortic amyloidosis is solely limited to the ATTRwt subtype, however no studies show a significant

287 occurrence of aortic involvement in $\mathrm{AL}$ amyloidosis ${ }^{26-28}$.

\section{5. Management and Prognosis}

289 Management of amyloidosis varies with each subtype; therefore, correct diagnosis is imperative ${ }^{7}$.

290 If incorrectly diagnosed, the prognosis can be far poorer because amyloidosis has varying causes

291 that do not respond to the same treatment ${ }^{7}$. One of the two main strategies used for managing

292 CA is to address cardiac symptoms and signs and improve stability ${ }^{8}$. Flow and conduction

293 abnormalities are common in CA, and traditional pharmacological treatments are generally

294 advised, however they may have adverse effects that are not properly investigated in patients

295 presenting with CA-related $\mathrm{HF}^{8}$. More evidence is needed to show the potential benefit of surgical 296 interventions and device therapy. The second strategy is to address the underlying cause of 297 cardiac symptoms by slowing the progression of amyloid deposition and is currently considered 298 the primary approach for managing $\mathrm{CA}^{8}$ and will therefore be the main focus of this section.

\section{$299 \quad 5.1$ Treating the cause of CA}

\subsubsection{AL amyloidosis management and prognosis:}


301 Patients with AL have a poor prognosis, and untreated median survival is 13 months ${ }^{7}$. Patients 302 with $A L$ and cardiac involvement have a very poor prognosis of 6-12 months after onset of 303 congestive cardiac failure ${ }^{5}$. Syncope, right ventricular dilatation, left ventricular wall thickness, and 304 elevated troponin I and T levels are all indicators of poor prognosis ${ }^{5}$.

305 Once diagnosed, AL can be treated with chemotherapeutic agents in combination with steroids ${ }^{7}$. 306 Standard treatment is cyclic oral melphalan and prednisone or dexamethasone, and the prognosis 307 is shown to be extended to 17 months $^{7}$. The 2015 British Committee for Standards in 308 Haematology Guideline recommends cyclophosphamide (CPA), bortezomib (BOR), and 309 dexamethasone (DEX) (together known as CyBorD or CVD), however treatment toxicity is 310 greater ${ }^{8}$. Recently, autologous peripheral blood stem-cell transplantation is suggested for far 311 better systemic outcomes, 40\% haematologic remission within a year, and prognosis extended to 3124.6 years $^{7}$. However, outcomes for patients with cardiac involvement remain low, with median 313 survival at 1.6 years, versus 5 months for the untreated patients ${ }^{7}$. The efficacy of thalidomide 314 combined with bortezomib has been shown and is expected to become more widely used in the 315 future $^{8}$.

316 Peri-transplant mortality rate has remained high for these patients, at $13 \%$. In patients with HF 317 due to CA, heart transplant remains the only option alongside palliative care ${ }^{29}$. Heart donations 318 are limited, and typically patients have a low chance of meeting the recipient criteria due to 319 systemic organ involvement of amyloidosis ${ }^{7}$. In a small cohort of 8 heart transplant recipients, 5 320 patients had evidence of amyloid deposits 5 months after transplantation ${ }^{7}$. This suggests that in 321 severe CA, the progression of the disease is not halted by only replacing the damaged organ. In 322 more recent data, heart transplants either with or without stem cell transplant, coupled with 323 aggressive chemotherapy to reduce the light chain replication, resulted in $100 \%$ one-year 324 survival ${ }^{5}$. Heart transplant remains a promising therapeutic option for cardiac amyloidosis, 325 particularly when systemic deposits are limited post-surgery with chemotherapy ${ }^{14}$. However, 5- 
13 | P a g e

year survival for patients undergoing heart transplant for cardiac amyloidosis was about half the survival rate for patients undergoing heart transplant for other indications ${ }^{14}$.

The most effective treatment of AL-CA remains early diagnosis and early drug treatment with a chemotherapeutic-steroidal combination.

\subsubsection{ATTR amyloidosis management and prognosis:}

The two main subtypes of ATTR (variant and wild-type), will be addressed together because of very similar pathogenesis and treatment ${ }^{8}$. Prognosis for cardiac-related ATTR amyloidosis (ATTR-CA) is better than for AL-CA, with median survival typically 2-6 years compared with 5-6 months ${ }^{7}$. Increasing amounts of evidence point to ATTR amyloidosis as a significant underlying cause of $\mathrm{HF}^{5-6,8}[5.6]$. High rates of misdiagnosis lead to harmful treatments, as many traditional treatments for cardiac disease are contra-indicated or of unknown or little benefit.

Treatment for AL-CA is not indicated in ATTR-CA, therefore correct diagnosis is necessary to avoid inappropriate chemotherapy ${ }^{30}$. The most effective treatment for ATTR amyloidosis remains liver transplant, as the liver produces the tetramers that misfold into amyloid proteins ${ }^{7-8}$. However, liver donations are not always available and some patients do not qualify for recipient criteria ${ }^{7}$. Furthermore, cardiac symptoms due to ATTR amyloidosis continue to progress after liver transplant, as well as retinal and cerebral deposits due to TTR production in the retinal epithelium and choroid plexus ${ }^{8}$.

Alternative or simultaneous (to liver transplant) treatment for ATTR amyloidosis includes drugs that inhibit liver TTR production, that stabilise TTR, and that increase excretion of TTR amyloid ${ }^{5}$. In 2012, Coehlo et al showed the efficacy of Tafamidis, a TTR stabiliser, for reducing all-cause mortality, cardiovascular disease hospitalisations and for improving quality of life ${ }^{8}$. Tafamidis has been shown to be beneficial for both ATTR-CA subtypes. Diflunisal is a non-steroidal anti- 
inflammatory drug (NSAID) with tetramer-stabilising properties, but further trials are needed to

350 prove its efficacy in light of typical NSAID adverse effects ${ }^{5}$. Other therapeutic agents recently

351 investigated are nucleic acids, which were effective in limiting the production of TTR ${ }^{8}$. Patisiran,

352 a small interfering RNA (siRNA) targeting TTR mRNA, reduced serum TTR by $80 \%$ after 18

353 months, and limited progression of cardiac signs ${ }^{8}$. Inotersen, an antisense oligonucleotide, was

354 also effective but had serious adverse effects ${ }^{7-8}$. Tafamidis and Patisiran are both recommended

355 as being potentially viable treatment options, but more trials are needed to prove their combined

356 efficacy or non-maleficence ${ }^{8}$. Doxycycline and (R)-1-[6-[(R)-2-carboxy-pyrrolidin-1-yl]-6-oxo-

357 hexanoyl]pyrrolidine-2-carboxylic acid (CPHPC) have been suggested due to their TTR amyloid-

358 clearing properties but further human trials are needed to prove efficacy ${ }^{5}$.

$359 \quad 5.2$ Aortic Stenosis (AS) in CA and its Prognosis:

360 CA may be more prevalent in elderly men with AS and is associated with far greater mortality.

361 The signs of TTR CA overlap considerably with low-flow AS from other causes, and $25 \%$ of post-

362 mortem hearts of octogenarians had amyloidosis (ATTRwt) deposits ${ }^{5}$. This suggests a great

363 proportion of undiagnosed CA, particularly in elderly patients. In another two studies, patients with

364 severe AS and treated with aortic valve replacement (AVR) were biopsied for amyloid deposits -

$36516 \%$ of which had CA in both studies, and in one study, $32 \%$ amongst the men only ${ }^{17,31}$. CA-AS

366 had a 3-fold greater mortality rate than AS alone in patients treated with AVR (56\% vs

$36720 \%)^{6}$. According to Java et al, AVR for other indications is not harmful in patients with

368 amyloidosis, and provides symptom relief in the mid-term ${ }^{32}$. However, prior evidence - which is

369 mostly on a handful of case studies - shows that prognosis of AVR in patients with amyloidosis is

370 a mixed picture ${ }^{32-33}$. There are particularly poor outcomes for entry at the apical site and better

371 outcomes for trans-femoral $\mathrm{AVR}^{32}$. Evidence analysed by Çiçek et al. suggests that AVR make

372 no improvement to the overall mortality of patients with $\mathrm{CA}^{33}$. Limitations to the study by Java et

373 al. is that due the small cohort of patients $(n=16)$, with possible selection bias, and that these 
patients have various subtypes of amyloidosis, therefore, the quality of this evidence is insufficient for a reliable recommendation for effective and sustainable treatment with AVR in patients with amyloidosis $^{32}$. In another paper, AVR as well as other cardiac surgery including remodelling, are strongly advised against based on the poor prognosis observed ${ }^{14}$, probably due to the surgery being non-curative for CA. Clinicians need to be wary of misdiagnosis of more common cardiac diseases in the presence of the ambiguous cardiac amyloidosis symptoms and signs ${ }^{14}$, or "probably vastly underrecognised concomitant amyloidosis"33.

381 Therefore, aortic valve replacement alone is not a suitable treatment for patients with AS caused 382 by CA. Thorough investigation is needed to confirm and appropriately treat the underlying cause of heart disease, which is more commonly due to amyloidosis in older patients. Appropriate treatment of amyloidosis will result in better survival outcomes, as opposed to cardiac surgery 385 alone.

\section{$\underline{\text { 6. Future research }}$}

387 There is need for evidence regarding the specific efficacy and potential adverse effects of 388 traditional HF medication, surgery and device therapy in relation to the management of symptoms 389 in patients with $\mathrm{CA}^{8}$. Additional further research also needs to be conducted in regard to 390 developing more accurate, non-invasive diagnostic techniques.

391 As mentioned above, Diflunisal has potential to be used in future treatment regimes, however, 392 randomised trials are needed to solidify its efficacy and especially weighing up its benefits 393 compared to common NSAID adverse effects that may be associated with its use ${ }^{5}$. Phase 3 trials 394 of Tafamidis have been completed further supporting its efficacy but despite this there is still a 395 need for a randomised-control trial to further consolidate the evidence supporting $\mathrm{it}^{34}$. As human 396 trials for both Doxycycline and CPHPC are in their infancy there is still a great need for further 397 research to be done on its use ${ }^{5}$. Focused research to further improve our understanding of the 
specific mechanism and factors of misfolding is necessary in order to develop more targeted

therapies.

\section{Conclusion}

401

402

403

404

405

406

407

408

409

410

411

412

413

414

415

416

417

418

419

420

421

422

423

424

425

426

427

428

429

430

431

432

Despite the rarity of amyloidosis and that it is still being underdiagnosed, the epidemiological data infers that the rate of diagnosis is improving most likely due to increased awareness of the disease and the development of more diagnostic techniques. The improvements in the diagnostic process should hopefully lead to earlier and more accurate detection of the disease which have shown to dramatically improve the management and prognosis of patients. Even with all these improvements there is still lack of evidence in our understanding of the cause of the disease as well as not enough evidence to support potential use of many of the proposed treatments. By addressing this dearth of evidence regarding amyloidosis, marked further improvements can be made towards better patient outcomes and their care.

\section{References}

1. Martinez-Naharro A, Hawkins PN, Fontana M. Cardiac amyloidosis. Clin Med (Lond). 2018;18(Suppl 2):s30-s35. doi:10.7861/clinmedicine.18-2-s30

2. Wechalekar AD, Gillmore JD, Hawkins PN. Systemic amyloidosis. Lancet. 2016 Jun 25;387(10038):2641-2654. doi: 10.1016/S0140-6736(15)01274-X.

3. Siddiqi OK, Ruberg FL. Cardiac amyloidosis: An update on pathophysiology, diagnosis, and treatment. Trends Cardiovasc Med. 2018;28(1):10-21. doi:10.1016/j.tcm.2017.07.004

4. Maleszewski JJ. Cardiac amyloidosis: pathology, nomenclature, and typing. Cardiovasc Pathol. 2015 Nov-Dec;24(6):343-50. doi: 10.1016/j.carpath.2015.07.008.

5. Dungu JN. Cardiac Amyloid - An Update. Eur Cardiol. 2015;10(2):113-117. doi:10.15420/ecr.2015.10.2.113

6. Cavalcante JL, Rijal S, Abdelkarim I, et al. Cardiac amyloidosis is prevalent in older patients with aortic stenosis and carries worse prognosis. J Cardiovasc Magn Reson. 2017;19(1):98. Published 2017 Dec 7. doi:10.1186/s12968-017-0415-x

7. Hassan W, Al-Sergani H, Mourad W, Tabbaa R. Amyloid heart disease. New frontiers and insights in pathophysiology, diagnosis, and management. Tex Heart Inst $J$. 2005;32(2):178-184.

8. Kitaoka H, Izumi C, Izumiya Y, Inomata T, Ueda M, Kubo T, Koyama J, Sano M, Sekijima Y, Tahara N, Tsukada N, Tsujita K, Tsutsui H, Tomita T, Amano M, Endo J, Okada A, Oda S, Takashio S, Baba Y, Misumi Y, Yazaki M, Anzai T, Ando Y, Isobe M, Kimura T, Fukuda K; Japanese Circulation Society Joint Working Group. JCS 2020 
Guideline on Diagnosis and Treatment of Cardiac Amyloidosis. Circ J. 2020 Aug 25;84(9):1610-1671. doi: 10.1253/circj.CJ-20-0110

9. Nienhuis HL, Bijzet J, Hazenberg BP. The Prevalence and Management of Systemic Amyloidosis in Western Countries. Kidney Dis (Basel). 2016;2(1):10-19. doi:10.1159/000444206

10. Falk RH. Diagnosis and management of the cardiac amyloidoses. Circulation. 2005;112(13):2047-2060. doi:10.1161/CIRCULATIONAHA.104.489187

11. Rowczenio DM, Noor I, Gillmore JD, et al. Online registry for mutations in hereditary amyloidosis including nomenclature recommendations. Hum Mutat. 2014;35(9):E2403E2412. doi:10.1002/humu.22619

12. Sekijima $Y$, Wiseman RL, Matteson J, et al. The biological and chemical basis for tissueselective amyloid disease. Cell. 2005;121(1):73-85. doi:10.1016/j.cell.2005.01.018

13. Falk RH, Dubrey SW. Amyloid heart disease [published correction appears in Prog Cardiovasc Dis. 2010 Mar-Apr;52(5):445-7]. Prog Cardiovasc Dis. 2010;52(4):347-361. doi:10.1016/j.pcad.2009.11.007

14. Zacek P, Harrer J. Amyloidosis and Cardiac Surgery. In: Narin C, editor. Perioperative Considerations in Cardiac Surgery [Internet]. InTech; 2012 [cited 2020 Sep 26]. Available from: http://www.intechopen.com/books/perioperative-considerations-incardiac-surgery/cardiac-amyloidosis-versus-cardiac-surgery

15. Manolis AS, Manolis AA, Manolis TA, Melita H. Cardiac amyloidosis: An underdiagnosed/underappreciated disease. Eur J Intern Med. 2019;67:1-13. doi:10.1016/j.ejim.2019.07.022

16. Gertz MA, Dispenzieri A, Sher T. Pathophysiology and treatment of cardiac amyloidosis. Nat Rev Cardiol. 2015;12(2):91-102. doi:10.1038/nrcardio.2014.165

17. Arenja N, Andre F, Riffel JH, Siepen FAD, Hegenbart U, Schönland S, Kristen AV, Katus HA, Buss SJ. Prognostic value of novel imaging parameters derived from standard cardiovascular magnetic resonance in high risk patients with systemic light chain amyloidosis. J Cardiovasc Magn Reson. 2019 Aug 22;21(1):53. doi: 10.1186/s12968019-0564-1

18. Dispenzieri A, Gertz MA, Kyle RA, et al. Serum cardiac troponins and N-terminal probrain natriuretic peptide: a staging system for primary systemic amyloidosis. J Clin Oncol. 2004;22(18):3751-3757. doi:10.1200/JCO.2004.03.029

19. Quock TP, Yan T, Chang E, Guthrie S, Broder MS. Epidemiology of AL amyloidosis: a real-world study using US claims data. Blood Adv. 2018 May 22;2(10):1046-1053. doi: 10.1182/bloodadvances.2018016402.

20. Lane T, Pinney JH, Gilbertson JA, et al. Changing epidemiology of AA amyloidosis: clinical observations over 25 years at a single national referral centre. Amyloid. 2017;24(3):162-166. doi:10.1080/13506129.2017.1342235

21. Modesto KM, Dispenzieri A, Gertz M, et al. Vascular abnormalities in primary amyloidosis. Eur Heart J. 2007;28(8):1019-1024. doi:10.1093/eurheartj/ehm066

22. Sharma PP, Payvar S, Litovsky SH. Histomorphometric analysis of intramyocardial vessels in primary and senile amyloidosis: epicardium versus endocardium. Cardiovasc Pathol. 2008;17(2):65-71. doi:10.1016/j.carpath.2007.05.008

23. Smith TJ, Kyle RA, Lie JT. Clinical significance of histopathologic patterns of cardiac amyloidosis. Mayo Clin Proc. 1984;59(8):547-555. doi:10.1016/s0025-6196(12)61493-1

24. Crotty TB, Li CY, Edwards WD, Suman VJ. Amyloidosis and endomyocardial biopsy: Correlation of extent and pattern of deposition with amyloid immunophenotype in 100 cases. Cardiovasc Pathol. 1995;4(1):39-42. doi:10.1016/1054-8807(94)00023-k

25. Dorbala S, Vangala $\mathrm{D}$, Bruyere $\mathrm{J} \mathrm{Jr}$, et al. Coronary microvascular dysfunction is related to abnormalities in myocardial structure and function in cardiac amyloidosis. JACC Heart Fail. 2014;2(4):358-367. doi:10.1016/j.jchf.2014.03.009 
26. Miura $\mathrm{Y}$, Tsumoto $\mathrm{H}$, Iwamoto $\mathrm{M}$, et al. Age-associated proteomic alterations in human aortic media. Geriatr Gerontol Int. 2019;19(10):1054-1062. doi:10.1111/ggi.13757

27. Iwata T, Kamei T, Uchino F, Mimaya H, Yanagaki T, Etoh H. Pathological study on amyloidosis--relationship of amyloid deposits in the aorta to aging. Acta Pathol Jpn. 1978;28(2):193-203. doi:10.1111/j.1440-1827.1978.tb00531.x

28. Enqvist S, Peng S, Persson A, Westermark P. Senile amyloidoses--diseases of increasing importance. Acta Histochem. 2003;105(4):377-378. doi:10.1078/0065-128100727

29. Yoon DW, Park BJ, Kim IS, Jeong DS. Isolated Tricuspid Regurgitation: Initial Manifestation of Cardiac Amyloidosis. Korean J Thorac Cardiovasc Surg. 2015;48(6):422-425. doi:10.5090/kjtcs.2015.48.6.422

30. O'Meara E, McDonald M, Chan M, et al. CCS/CHFS Heart Failure Guidelines: Clinical Trial Update on Functional Mitral Regurgitation, SGLT2 Inhibitors, ARNI in HFpEF, and Tafamidis in Amyloidosis. Can J Cardiol. 2020;36(2):159-169. doi:10.1016/j.cjca.2019.11.036

31. Castaño A, Narotsky DL, Hamid N, et al. Unveiling transthyretin cardiac amyloidosis and its predictors among elderly patients with severe aortic stenosis undergoing transcatheter aortic valve replacement. Eur Heart J. 2017;38(38):2879-2887. doi:10.1093/eurheartj/ehx350

32. Java AP, Greason KL, Dispenzieri A, et al. Aortic valve replacement in patients with amyloidosis. J Thorac Cardiovasc Surg. 2018;156(1):98-103. doi:10.1016/j.jtcvs.2017.12.048

33. Çiçek S. Cardiac amyloidosis and aortic stenosis: Great masquerader back again!. J Thorac Cardiovasc Surg. 2018;156(1):104-105. doi:10.1016/j.jtcvs.2018.01.080

34. Maurer MS, Schwartz JH, Gundapaneni B, et al. Tafamidis Treatment for Patients with Transthyretin Amyloid Cardiomyopathy. N Engl J Med. 2018;379(11):1007-1016. doi:10.1056/NEJMoa1805689 